\title{
Adaptive White-fi Spectrum Channel Deployment Intended to Configurable Femtocell Network
}

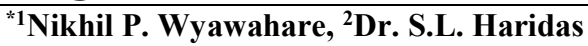 \\ ${ }^{*}$ Research Scholar, IEEE Member, Department of Electronics Engineering \\ ${ }^{2}$ Professor, IEEE Member Department of Electronics \& Telecommunication Engineering \\ ${ }^{1,2}$ G.H.Raisoni College of Engineering, Nagpur, India \\ Email:nikhil.wyawahare@raisoni.net,sanjay.haridas@raisoni.net
}

Received: $13^{\text {th }}$ December 2018, Accepted: $13^{\text {th }}$ February 2019, Published: $30^{\text {th }}$ June $_{2019}$

\begin{abstract}
In this paper an adaptive white-fi spectrum deployment of a Femtocell as small scale private network for home and office is intended. Its design is underlay to utilized white-spaces available from empty or partial empty channels typically received RSSI $>-70 \mathrm{~dB}$ above strength of the white-fi spectrums. Core key is to establish service in between local to another local user's which works on the available Wi-Fi network. Here the Role of local user to communicate between all other users, likely the others too. Since the local user is using its mobile or cell phone then the network for the local user is of two type's primary and secondary, in which he is one the user who comes under license spectrum range and actually he buys the network from the service provider. The point of study is when frequency is getting jammed or out of coverage due to this the local user will unable to communicate in primary network as fact. On contradictory he has another network which can easily serve him. Looking to this a white-fi network which is design for small private network a Femtocell is designed. This network is temporary replacement of the primary one where the local user is comes in set of big primary network and under it can also falls in secondary network. The white-fi network is working on the dynamic frequency scanning, where a base station is small cognitive network to find free channels out of available for to resist the co-channel interference and the overlapping could be minimized. The detection of the signal in all noise channels is done through the passive and active channel scanning, along with the hybrid scanning technique min-max algorithms and feature detection. The identified channel is deployed only for secondary network node where the same user will communicated where primary network is not serving. Since use of available frequency is part of network and from which only 1,6,11 channel are rigorously being utilized due to normal scanning, all other channel mostly channel which are remains empty like 14 no. can be utilized for such a user who can communicate with its same device. The part of implementation of this work is scanning of ISM band spectrum channel 2.4 to $2.484 \mathrm{GHz}$ where FAP (Femtocell access point) is Linux base station where the active scanning uses as OSA and DSA method. Use of MIMO Signalling scheme for DTE section is implemented.
\end{abstract}

\section{Keywords}

FAP-Femtocell-Small Network for Home/Office, White-fi- White-Space, OSA-Opportunistic Spectrum Access, DSA-Dynamic Spectrum Access, MIMO- Multi Input Multi Output, DTE- Digital Terminal Equipment.

\section{Introduction}

Wireless communication is the relocating or distribution of the information from one point to many points, where connection orientation is wired or wireless. In modern case Wireless technology used everywhere, for short and effective distance communication [1] as well as long distance communication. A most common example of this wireless technology are Mobile communication, wireless router, GPS, Broadcast of television, Cordless telephone, wireless music systems and many more. All these technologies where work on dedicated range of channel specially allocated in their regions only to separate mode of application. As simple facts if all these channels when packed due to heavy data packets, generally then tends to have overlapping as transmitting channel or band is always band limited. Many of such cases are commonly called as "Intersymbol Interference" due to which a co-channel existence. These ISI are never to be removed from the transmission error, are only minimized by applying the correlative encoding and methods where the duplication of the data bits are done on the specific time duration as called $\mathrm{Tb}$. thus for we have treated Intersymbol interference as an undesirable phenomenon that produces a degradation in system performance. Indeed, its very name can also note a nuisance effect. 


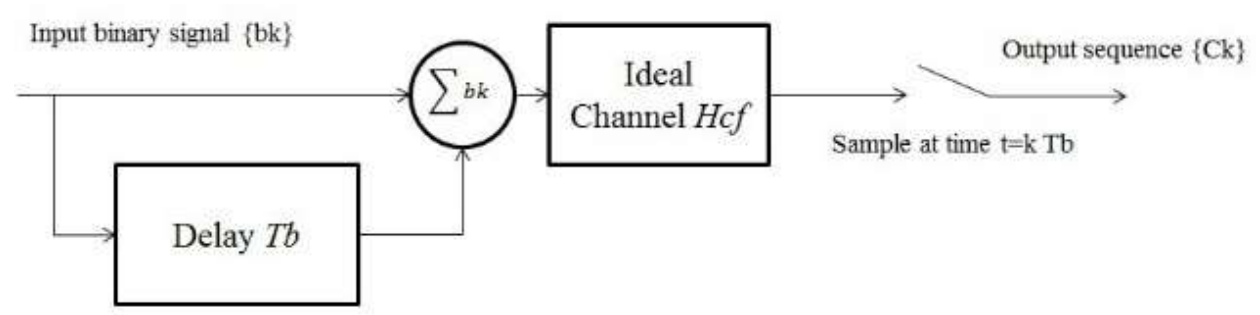

Figure 1: Duo Binary Signalling Scheme

Nevertheless, by adding Intersymbol interference to the transmitted signal in a controlled manner, it's possible to achieve a bit rate of $2 \mathrm{Bo}$ bits per second in a channel of bandwidth Bo Hz. Scheme likely to be term partial response signalling. The basic design of correlative coding will base on due word stands for the doubling of the transmission capacity of a straight binary system. In which a binary sequence $\{b k\}$ consisting of uncorrelated binary digits each having duration $\mathrm{Tb}$ Seconds, with symbol ' 1 ' represented by a pulse of amplitude $+1 \mathrm{v}$ and symbol ' 0 ' by a pulse of amplitude $-1 \mathrm{v}$. When this sequence is applied to an encoder it is converted in to threelevel output, viz. $-2,0,+2$ volts. Illustrated in correlative signalling system. The duo binary sequence $\{\mathrm{bk}\}$ is passed a filter involving as single delay element for every impulses from train of pulses. From here we get two units impulse spaced $\mathrm{Tb}$ seconds apart at the filter output. It is express the digit $\mathrm{Ck}$ at the duo binary coder output as the Sum of the present binary digit bk and its previous values bk-1 as given like $\mathrm{Ck}=\mathrm{bk}+\mathrm{bk}-1$ which shows the effect of transformation tc change the input sequence $\{\mathrm{bk}\}$ of uncorrelated binary digits in to a sequence $\{\mathrm{Ck}\}$ of correlated digits. Actually this correlation between the adjacent transformation levels may be viewed as introduction of Intersymbol interference [ISI] this may be a co-channel too will be refer to insert in artificial manner. However all this is under designer control.

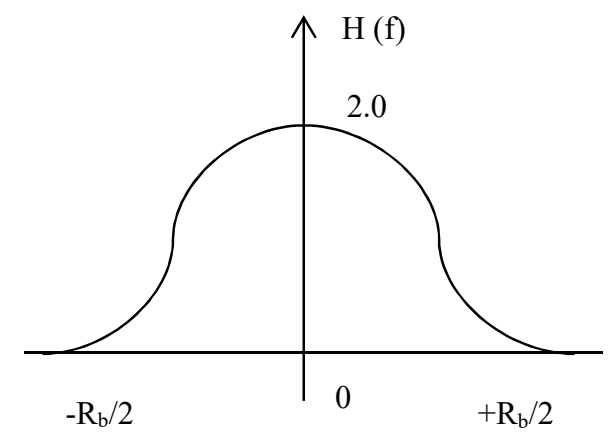

Figure 2: Amplitude Response for Signalling

If the ideal channel is consider with bandwidth $\mathrm{B} 0=\mathrm{Rb} / 2$ then we have, $\quad \mathrm{Hc}$ (f) $\left\{\begin{array}{cc}1|f|>\frac{R b}{2} \\ \text { limit } \\ 0 \text { otherwise }\end{array}\right.$

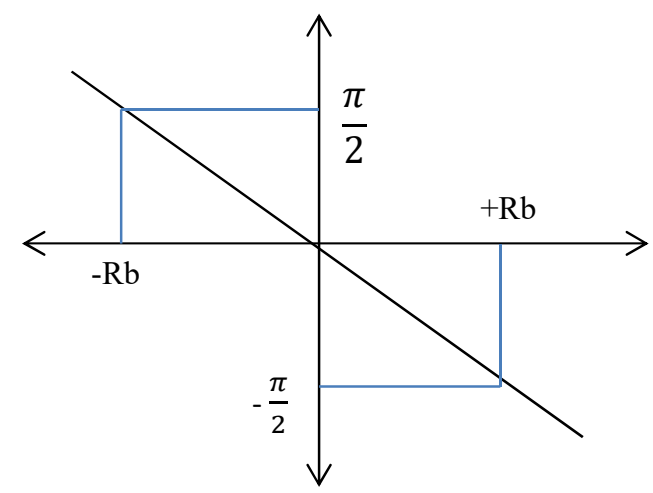

Figure 3: Phase Response

From the this detection techniques a simple filter will be observed at the transmitter, however a drawback of this system is that ones the errors are maid they tends to propagate due to this fact a decision on the current binary made on the previous binary digit bk-1. A practical means of avoiding this errors propagation is to use precoding before the duo binary coding. 


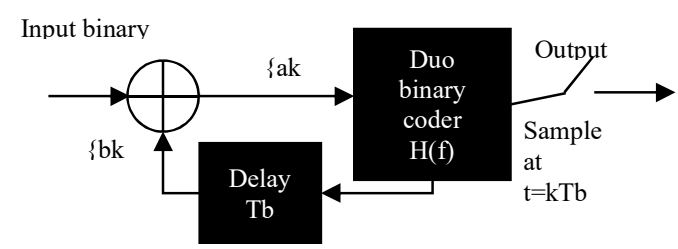

Design \& Implementation:

Figure 4: A Proceeded Duo Binary Scheme

Using the above scheme Intersymbol interference can be removed and data is getting secured in channel. Ones the data is secured there is need to scan it and utilize to have secondary communication in the Femtocell network. As following section is introducing the $2.4 \mathrm{GHz}$ band scanner. It is first prototype with Mat lab base scanner hardware interfacing, [5] the implementation of real time RF scanner hardware is divided in mainly three block i.e. RF module of CY8CPLC2050,[2] Computer and MATLAB software. The first block is a RF module of Cypress named CY8CPLC2050 which consists of Microcontroller, a voltage regulator and a patch antenna. A voltage regulator is used to give a constant voltage of $3.3 \mathrm{~V}$ to the RF module as this module work on $3.3 \mathrm{~V}$.Voltage regulator IC used here in this module is AMS1117. The antenna used here is patch antenna especially for $2.412-2.483 \mathrm{GHz}$. Second block is a computer which act as an intermediator. Here all the preprocessing work of the entire project related work has been done. Handling allude to all the strides require to give the info require to the MATLAB programming. MATLAB programming is utilized to the different diagram identified with power and SNR proportion. Here using Energy detection method spectrum scanning is performed .Energy detection method is based on the threshold set for power. Here first of all at threshold of power is set for scanned spectrum. After setting the threshold for the procedure scanned spectrum scanning of frequency spectrum is started. After the scanning of frequency spectrum, power and SNR ratio of scanned spectrum is calculated .Comparison of power of the scanned spectrum is done with pre-set threshold. After comparison of the power scanned spectrum with the set threshold availability of the vacant channel is determine. If power of scanned spectrum is less than the set threshold then the channel is vacant otherwise it is busy. For the scanning, here Min- Max algorithm is implemented which is illustrated in following figure 5.

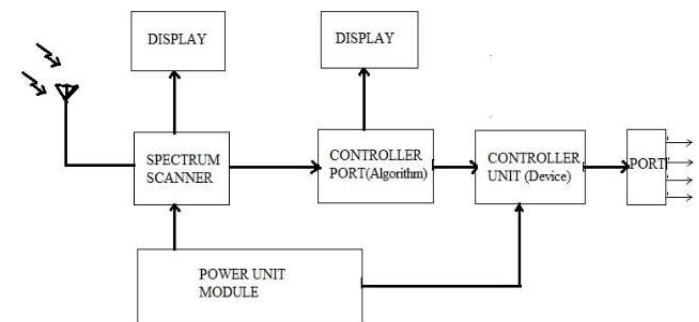

Figure 5: Min- Max Algorithm for Power Ratio Optimization

1. Spectrum Scanner: This part of the block diagram contains the spectrum scanner which is used to scan the spectrum by sensing it through a RF Module named CY8CPLC2050 .The RF module consist of a [3] patch antenna, a microcontroller and voltage regulator.

2. Controller Port (Algorithm): This part is mainly consisting of an algorithm named MIN-MAX algorithm. This algorithm gathered the information sensed by spectrum scanner. Based on this phase, magnitude of the spectrum is determined .Based on this values probability, power and SNR ratio is calculated. On the basis of these values availability of vacant channel is determined.

3. Controller Port (Device): This part involve the controlling of antenna module which are waiting for transmitting data over the available channel .Based on the results of power and SNR controlling of antenna module is done.

4. Power Unit Module: This unit is used to plot various graphs of power of scanned spectrum which will give the information regarding the availability of vacant channel. This will have some limitation related to use of controller, there is need of fast operating system to operate on dynamic mode of scanning. Thus cypress CYWM6935 scanner is used to implement using microcontroller base so that faster and more port will there for switching unit interface. 


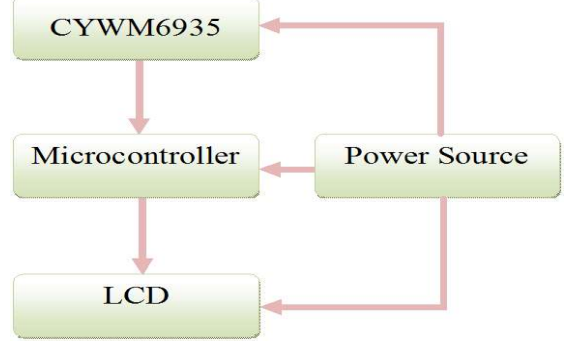

Figure 6: CYWM6935 Portable White Space Scanner

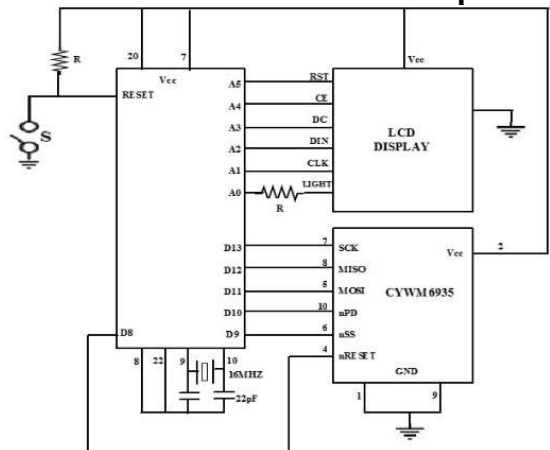

Figure 7: Interface for CYWM6935 Scanner

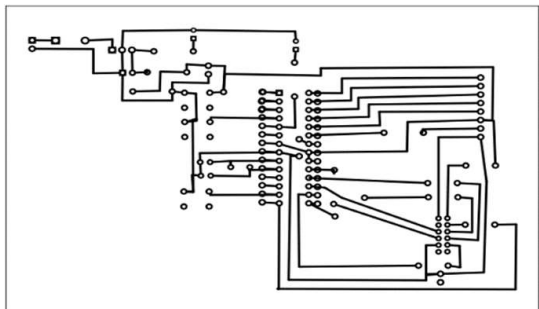

Figure 8: PCB Layout for CYWM6935 Scanner

Figure 8 shows the implementation of circuit on PCB, the most useful tool i.e. Fritzing is used. Fritzing a software program to help designers translate their prototypes into real implementation. Created at the University of Applied Sciences, Potsdam, and the software is an open source software tool. It runs on Linux, Mac OS X and MS Windows. In Fritzing software we can implement the circuit on three ways i.e. either on bread board or schematic or directly PCB design, as illustrated in figure 8. PCB layout design for CYWM6935 this can be a printed using the Fritzing software on glossy photographic paper using a laser printer. Using Electronics Workshop lab it is easily fabricated on to copper clad after etching process. Illustrated in Figure 10. Here [4] the CYWM6935 Spectrum sensing prototype working module hardware consists of Microcontroller, CYWM6935 Transceiver, Serial Display and Power Supply. The microcontroller is ATmega328 that will have coding that is in Embedded C and it can program by the Arduino software. The CYWM6935 is the transceiver module manufactured by Cypress.

Here [4] the CYWM6935 Spectrum sensing prototype working module hardware consists of Microcontroller, CYWM6935 Transceiver, Serial Display and Power Supply. The microcontroller is ATmega328 that will have coding that is in Embedded C and it can program by the Arduino software. The CYWM6935 is the transceiver module manufactured by Cypress. The job and scope of the radio module is to conducts a test to verify device's ability to detect ISM signal. And it is [5] control by the microcontroller using SPI interface. The serial display is the graphical LCD of the Nokia 5110 phone. It feature is that it can run the serial data- ensures that it will be able to display the spectrum detected. Power source will implement the power system that has capability in providing 2.7 to $3.3 \mathrm{~V}$ and required current to each module in the whole board. From ISM band the real time data is to be sensing by the receiver module. The microcontroller is programmed such that radio module detects the signal level and that signal level is display on the serial display. Microcontroller is program by the Arduino software and the Whole circuit is design on Fritzing software for printing the PCB. From the above design work still it need to interface with switching unit so that relay unit will give the proper channel to communicate in between SU client. Here Cypress [7] CYWM6935 module it is interfaced with switching unit and GUI interface is created in PC to observe the scanning information. Using the relay all FAP points will be 
getting access to scanned free white spaces, these scanning is done in Active and Passive mode for initial and dynamic way.

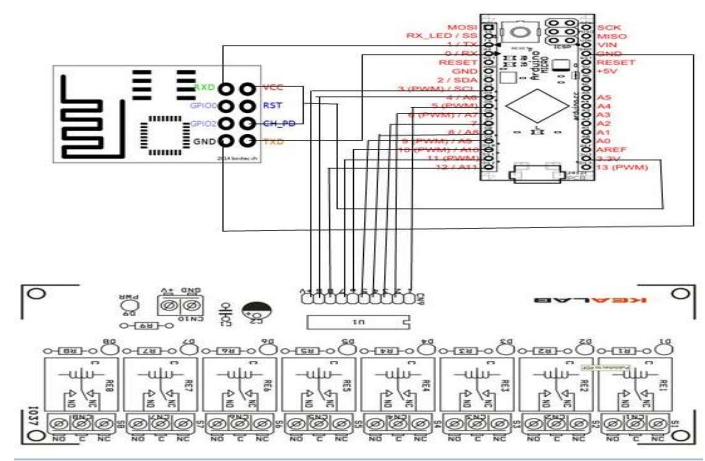

Figure 9: Advance White Space Scanner System

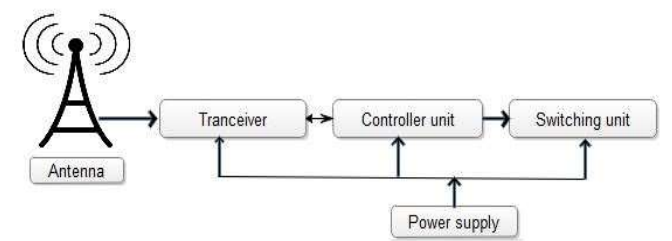

Figure 10: Proposed Cypress Scanner Module

This design [6] working as the cognitive base in the following flow structure. [9] The detail working flow process of cognitive white space scanner unit, where the controller is started with initial scanning to dynamic scanning. In 2.412 to $2.484 \mathrm{GHz}$ band of total 14 channels are getting scanned and displayed on PC GUI. After detecting the channel in busy-partial busy-free threshold level checking in $\mathrm{dB}$ range it is signaled to switching unit to control Femtocell access point to establish secondary network.

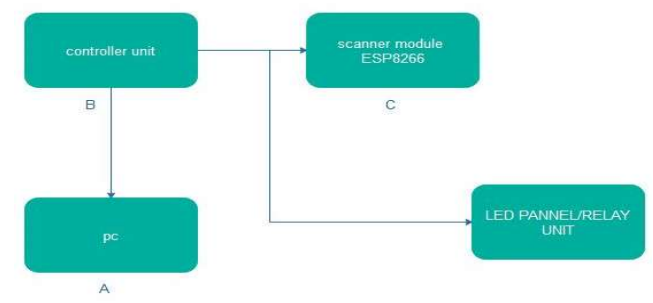

\section{Results}

Figure 11: Process Flow of Cognitive White Space Switching Unit

From the previous result \& objective [5] now there is need of robust scanning of all ISM band option those who are proving as communication network. Depending upon the way of scanning following scanner with use of PC controller where used to find out Figure 12 shows result according to threshold which was set 0.5 the channels in block 1 are having probability value greater than 0.5 so channel are free.

Comparison in between the SNR to power ratio. This will be the first design prototype to observed scanned output in different channel wise.

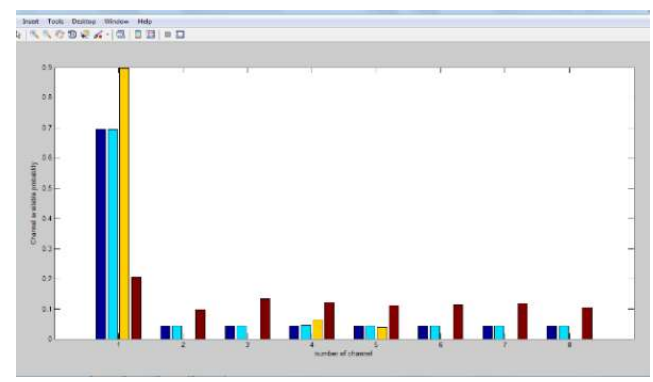

Figure 12: Probability Calculated Over Scanned Spectrum for Channel 1 
Figure 12 illustrating, the above figures state the probability of various scanned spectrum. During spectrum scanning output power is calculated. As it told earlier that a preset threshold for power is set using energy detection method for spectrum sensing. Depending upon the power calculated of the scanned spectrum availability of channel is determined .If power of scanned spectrum is less than preset power then channel is available for transmission. The above result shows the availability of the vacant channel in the form of bar graph. [8] The main principle behind this is that depend on the set threshold value the availability of the channel in the frequency spectrum. Energy Detection Method is a method in which spectrum sensing is done on the basis of set threshold value .Comparison of power we get in spectrum sensing is done with the prior set threshold value .Based on this we got various bar graph which showing availability of vacant channel. The results of spectrum measurements had been presented in the ISM [9] band prepared at three diverse places in the Nagpur area. One location is at the highly dense region in urban place. Another location is the semi urban area. A third location is in the outside of Nagpur, in a rural place. At each spectrum chart that shows variations in time and places, an economical spectrum sensing module has developed which used in White Space Networks.

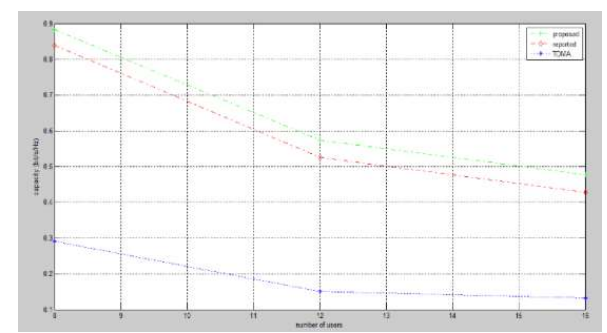

Figure 13: Channel Capacity Vs. Number of Users in TDMA Scanning

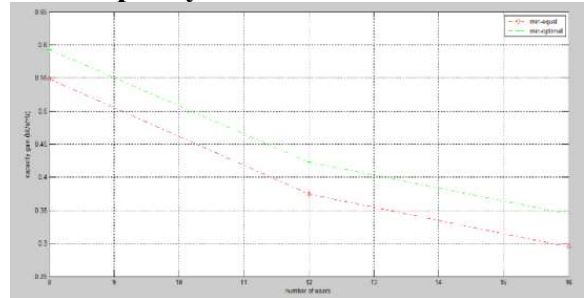

Figure 14: Channel Capacity in Spectrum Scanning Using Min-Max Algorithm

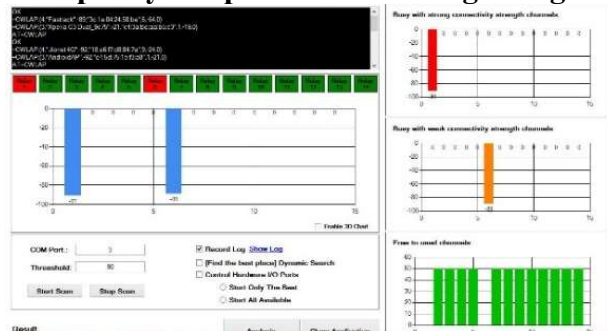

Figure 15: Advance Scanning of Spectrum for Channel $1 \& 6$

Figure 15 shows scanning [8] results and bar graph only shows busy channel number but in advance to that result generated by busy channel with strong connectivity strength and channel busy with weak connectivity and free available channels to use in real time by cognitive White space scanner. And results come out after scanning other than Femtocell network illustrated in figure 16 shows the massive change in the channel no utilization. This scanning is dynamic in nature depending upon this cognitive white space scanner is taking decision on channel with -70dB RSSI having week signal or -50dB having strong signal.

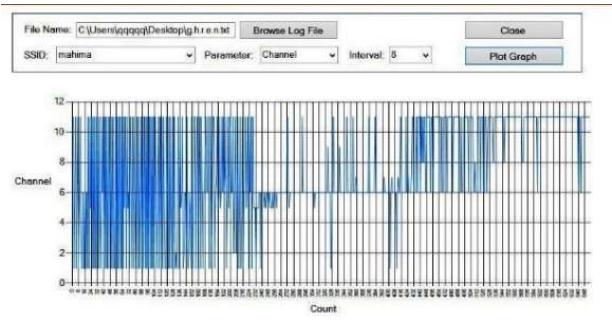

Figure 16: Scanning of "Mahima" SSID for Channel Vs. Count 


\section{Conclusion}

Following design and implementation is scanning the ISM band spectrum from 2.412 to $2.483 \mathrm{GHz}$, in which only channel 14 are available to use for to communicate. These channels are mostly channel $1,6 \& 11$, act as dominating channels. At the time of communication there always co channels interference may reduce the speed of data rate, hence using a doubling of data it will reduce the cause of error. After that scanning of channels will be have some meaning to use for the data to transmit. Thus the scanning is in mode of Active and Passive in mode, here initial and dynamic scanning is implemented to have the real time scanning of channels from 1 to 14 channels with $20 \mathrm{MHz}$ band gap. In implementation of white space scanner is mostly required fast and dynamic scanning. After these fruitful scanning it's to be found out which channel is free and which one is busy. The free channel here called as white space so the designed implementation can be access all channel with full strength for communication services aspect. Then this will easy step of deployment of configurable Femtocell network.

\section{References}

1. Jaeweon Kim, Student Member, IEEE, and Jeffrey G. Andrews, Senior Member, IEEE, "Sensitive White Space Detection with Spectral Covariance Sensing" in IEEE TRANSACTIONS ON WIRELESS COMMUNICATIONS, ACCEPTED FOR PUBLICATION, 2010.

2. Peter Flynn, Product and program manager, Multicore processos Texas Instruments, "White SpacePotentials and Realities", January 2013.

3. Jaap VAN DE BEEK, Janne RIIHIJ ARVI, Petri MAHONEN, "Intrinsic challenges (and opportunities) to deploy LTE in Europe's TV white spaces" in Future Network and Mobile Summit 2012 Conference Proceedings, Paul Cunningham and Miriam Cunningham (Eds) IIMC International Information Management Corporation, 2012

4. Farzad Hessar, Student Member, IEEE, Sumit Roy, Fellow, IEEE, “Capacity Considerations for Secondary Networks in TV White Space"arXiv:1304.1785v1 [cs.NI] 28 Mar 2013.

5. Kazushi Muraoka Hiroto Sugahara Masayuki Ariyoshi, "Monitoring-Based Spectrum Management for Expanding Opportunities of White Space Utilization", in 2011 IEEE International Symposium on Dynamic Spectrum Access Networks (DySPAN).

6. Mauro Fadda, Maurizio Murroni and Vlad Popescu, Member, IEEE, "A Cognitive Radio Indoor HDTV Multi-Vision System in the TV White Spaces", in IEEE Transactions on Consumer Electronics, Vol. 58, No. 2, May 2012

7. John Sydor, David Roberts, Bernard Doray and Amir Ghasemi, “A Generic Cognitive Radio Based on Commodity Hardware”, in IEEE INFOCOM 2011 Workshop On Cognitive \& Cooperative Networks

8. Manish Kumar Dhaka, Pankaj Verma "A Relay Based Cooperative Spectrum Sensing Selecting Maximum Value of SNR in Multi-Channel Cognitive Radio," IEEE International Conference on Recent Advances and Innovations in Engineering (ICRAIE-2014), May 09-11, 2014, Jaipur, India.

9. Narjes Torabi, Shantanu Bhate and Victor C.M. Leung, "Robust Sensing Strategy for Dynamic Spectrum Access in the $2.4 \mathrm{GHz}$ ISM Band," 2013 IEEE 24th International Symposium on Personal, Indoor and Mobile Radio Communications: Mobile and Wireless Networks.

10. Analysis of the FCC's Positions, Policies and Comments on Secondary Markets http: //Principles for Promoting the Efficient Use of Spectrum by Encouraging the Development of Secondary Markets, Policy Statement (FCC 00-401)

11. Wyawahare NP, Haridas SL. Designing challenges to uti lize white-space for configurable femtocell base on slotted TDM. IEEE, International Conference on Contemporary Computing and Informatics (IC3I); 2014. p. 1076-80. Crossref.

12. Nikhil P. Wyawahare, S. L. Haridas, "A Novel OSA based ISM Band Channel Scanner Design for Identification and Deployment of White-Spaces" Indian Journal of Science and Technology, Vol 10(43), DOI: $10.17485 /$ ijst/2017/v10i43/115338, November 201, ISSN (Print) : 0974-6846, ISSN (Online) : 09745645 